\title{
FSS-based approach for the power transmission enhancement through electrically small apertures
}

\author{
Luca Scorrano • Filiberto Bilotti · Ekmel Ozbay • \\ Lucio Vegni
}

Received: 30 March 2010 / Accepted: 3 December 2010 / Published online: 20 January 2011

(C) Springer-Verlag 2011

\begin{abstract}
In this paper, a novel approach, based on the employment of frequency selective surfaces, to enhance the power transmission through sub-wavelength apertures at the microwave frequencies is presented. A heuristic interpretation of the phenomenon is given, as well as an analytical model, based on the transmission line network representation. Finally, the performance of the proposed structure is validated through a set of full-wave numerical simulations.
\end{abstract}

\section{Introduction}

At the end of the 1990s, Ebbesen's group successfully demonstrated the possibility to overcome the diffraction limit at the visible frequencies by using properly designed periodic corrugations in metal screens with nanoscale holes [1]. This phenomenon, known as Extraordinary Optical Transmission (EOT), has been elegantly explained by Oliner and Jackson, in terms of the excitation of leaky surface plasmons coupling to the aperture [2]. During the last years, the strong interest in the EOT, as well as the introduction of new artificial materials (i.e. metamaterials-MTMs), led to the idea of scaling this phenomenon down to the microwave frequencies.

To the authors' best knowledge, the first setup proposed to accomplish this task has been presented in [3], based on

L. Scorrano $(\bowtie) \cdot$ F. Bilotti $\cdot$ L. Vegni

Department of Applied Electronics, University "Roma Tre",

Via della Vasca Navale, 84, 00146 Rome, Italy

e-mail: 1scorrano@uniroma3.it

E. Ozbay

Nanotechnology Research Center, Bilkent University, Bilkent, 06800 Ankara, Turkey properly designed MTM covers with Near-Zero (NZ) constitutive parameters and supporting proper leaky modes. However, the actual excitation of such modes, turned out to be rather challenging, due to the small imaginary part of the corresponding propagation constant. Another issue was the thickness of the MTM slab which is electrically large, being the index of refraction extremely low. As a consequence, the setup presented in [3] has not been practically implemented yet. Therefore, in order to foresee a possible actual implementation of this setup, we focused our effort in implementing the same physical mechanism by using a different approach, based on a frequency-selective surface (FSS), and placed in front of the perforated metallic plate.

\section{THE proposed setup}

According to the classical theory by Bethe [4], neglecting the retardation effects due to the thickness of the screen and assuming that the screen is perfectly conducting, the transmission through an electrically small hole can be described in terms of an equivalent electric dipole moment (orthogonal to the screen) with amplitude $p_{e}$ and an equivalent magnetic dipole moment (parallel to the screen) with amplitude $p_{m}$ :

$p_{e}=-\frac{1}{3 \pi} r^{3} E_{0}, \quad p_{m}=-\frac{2}{3 \pi} r^{3} H_{0}$

being $E_{0}$ and $H_{0}$ the electric and magnetic field amplitudes on the hole and $r$ the hole radius. Our idea is to design an FSS-based screen, which has been shown to be a valid alternative to replace bulk MTMs [5], whose complex surface impedance $\left(Z_{\mathrm{FSS}}\right)[6]$ is such that the amplitude of the equivalent magnetic dipole moment of the aperture results dramatically enhanced when the FSS is placed in front of the perforated metallic screen. The sketch of the proposed 


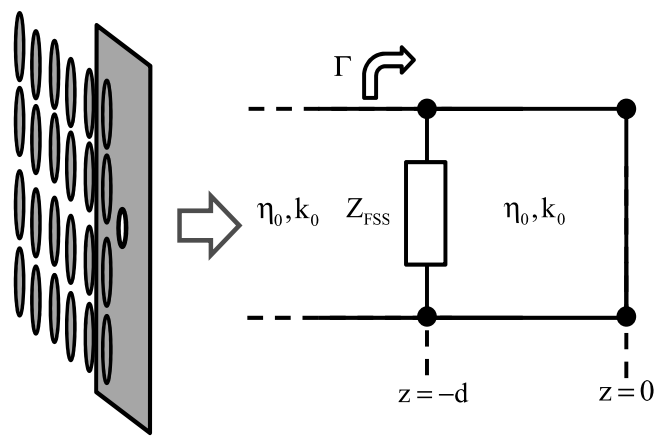

Fig. 1 Layout of the proposed setup (the disk shape of the FSS unit cell is used as a mere example) and the corresponding equivalent circuit representation. In the TL representation, the FSS is modeled through its surface impedance and the hole contribution has been neglected, due to its sub-wavelength dimensions

setup is reported in Fig. 1, along with its transmission line (TL) equivalent model.

\section{Design of the FSS based setup}

Aiming at enhancing the power transmission through the sub-wavelength aperture, the FSS surface impedance $Z_{\mathrm{FSS}}$ should be designed to increase the tangential magnetic field amplitude on the screen. The value of the equivalent shunt impedance of the FSS is derived as follows. Let us consider a monochromatic $\mathrm{TE}(z)$ plane wave excitation (the TM case can be easily obtained by duality) in the form:

$\mathbf{E}^{\mathrm{TE}}=\hat{x} E_{0}\left(e^{-j k_{z}(z+d)}+R^{\mathrm{TE}} e^{j k_{z}(z+d)}\right) e^{-j k_{y} y}$

$\mathbf{H}^{\mathrm{TE}}=\frac{j}{k_{0} Z_{0}} \nabla \times \mathbf{E}^{\mathrm{TE}}$

where $k_{0}=\omega \sqrt{\varepsilon_{0} \mu_{0}}, k_{y}=k_{0} \sin \theta_{i}, k_{z}=\sqrt{k_{0}^{2}-k_{y}^{2}}$. Exploiting the equivalent circuit representation depicted in Fig. 1, the tangential magnetic field on the perfectly conducting screen can be easily obtained as

$$
\begin{aligned}
\left.\mathbf{H}_{y}^{\mathrm{TE}}\right|_{z=0} \\
=\hat{y} \frac{2 E_{0} e^{-j k_{y} y} k_{z}^{2}}{k_{0} Z_{0}} \\
\quad \times \frac{Z_{\mathrm{FSS}}}{k_{z} Z_{\mathrm{FSS}} \cos \left(k_{z} d\right)+j\left(k_{z} Z_{\mathrm{FSS}}+k_{0} Z_{0}\right) \sin \left(k_{z} d\right)}
\end{aligned}
$$

Requiring the denominator to vanish on the screen, the following design formula is straightforwardly derived:

$$
Z_{\mathrm{FSS}}^{\mathrm{TE}}=-\left(k_{0} / k_{z}\right) Z_{0} \sin \left(k_{z} d\right)\left[j \cos \left(k_{z} d\right)+\sin \left(k_{z} d\right)\right]
$$

The real part of (4) is always negative, in contrast with the supposed passivity of the FSS. This means that, in general, the denominator cannot vanish exactly. However, under the assumption that the spacing between the FSS and the screen is electrically small $\left(k_{z} d \ll 1\right)$, a first order Taylor expansion of (4) gives

$Z_{\mathrm{FSS}}^{\mathrm{TE}}=-d\left(j+k_{z} d\right) k_{0} Z_{0} \simeq-j k_{0} Z_{0} d$

In the same way, for an incident $\operatorname{TM}(z)$ plane wave the following design formula is obtained:

$Z_{\mathrm{FSS}}^{\mathrm{TM}}=-\left(k_{z} / k_{0}\right) Z_{0} \sin \left(k_{z} d\right)\left[j \cos \left(k_{z} d\right)+\sin \left(k_{z} d\right)\right]$

Applying, again, a first order Taylor expansion, expression (6) becomes

$Z_{\mathrm{FSS}}^{\mathrm{TM}} \simeq-j Z_{0}\left(k_{z}^{2} / k_{0}\right) d$

Using either (5) or (7), it is possible to design, for a given frequency and distance $d$ between the FSS and the screen, the needed reactive impedance value leading to an increased amplitude of the tangential magnetic field on the aperture plane. A clear example is reported in Fig. 2(a), where the plot of the enhancement of the tangential magnetic field amplitude on the screen, normalized to the case without the FSS, is shown.

In a real-life application employing a passive FSS, the negative, yet small, value of the real part of $Z_{\mathrm{FSS}}$ has to be neglected, affecting thus the enhancement performance of the setup (Fig. 2(b)). However, even if the real part of the surface impedance is completely neglected (which is the actual case), the amplitude of the tangential magnetic field is highly enhanced anyway (around $10 \mathrm{~dB}$ with respect to the case of the screen alone without the FSS). A simple actual implementation can be based on the patterned squared loop metallic surface represented in Fig. 3(a), designed to exhibit the required reactive value of $Z_{\mathrm{FSS}}$ at $f_{0}=15 \mathrm{GHz}$.

This is an extremely simplified setup, designed to verify the reliability of the proposed approach. The angular bandwidth of this configuration is, in fact, mainly limited by the FSS unit-cell shape, being the distance $d$ electrically small. Further improvements are expected using more complex designs of the base element shape and a triangular lattice with circular shaped element of smaller size [6]. The structure shown in Fig. 3(a) has been simulated through CST Studio Suite [7] inside a parallel-plate waveguide, bounded by two perfect magnetic boundaries on the right/left sides in order to excite the $\operatorname{TEM}(z)$ fundamental mode. In the inset of Fig. 3(b), the variation of the magnetic field amplitude at the design frequency along the propagation direction passing through the hole, is reported. The expected strong magnetic field resonance is visible between the FSS and the metallic screen, where the hole is placed. The power transmission enhancement is close to $20 \mathrm{~dB}$ (Fig. 3(b)). 


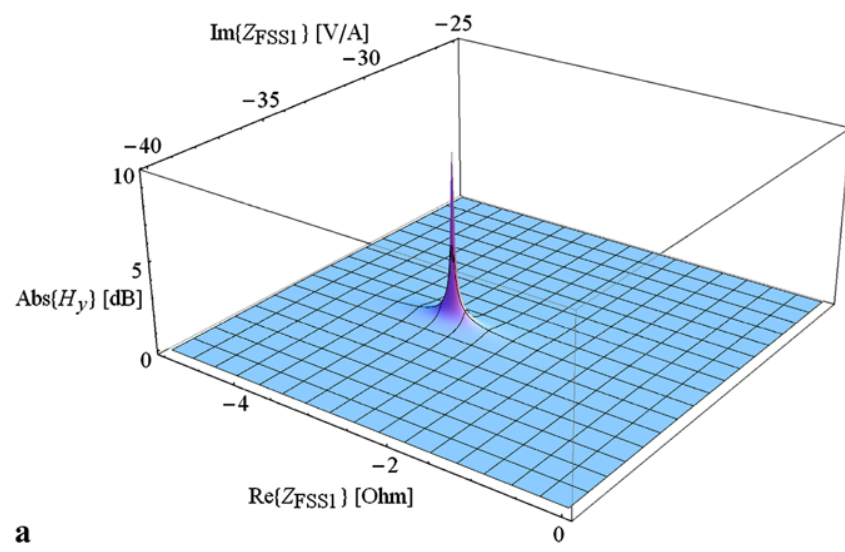

Fig. 2 (a) Amplitude in [dB] of the tangential magnetic field on the metallic screen (normalized to the case of absence of the FSS) as a function of the complex values of the shunt impedance of the FSS for a design frequency $f_{0}=15 \mathrm{GHz}$ and $d=0.25 \mathrm{~mm}$. The enhancement

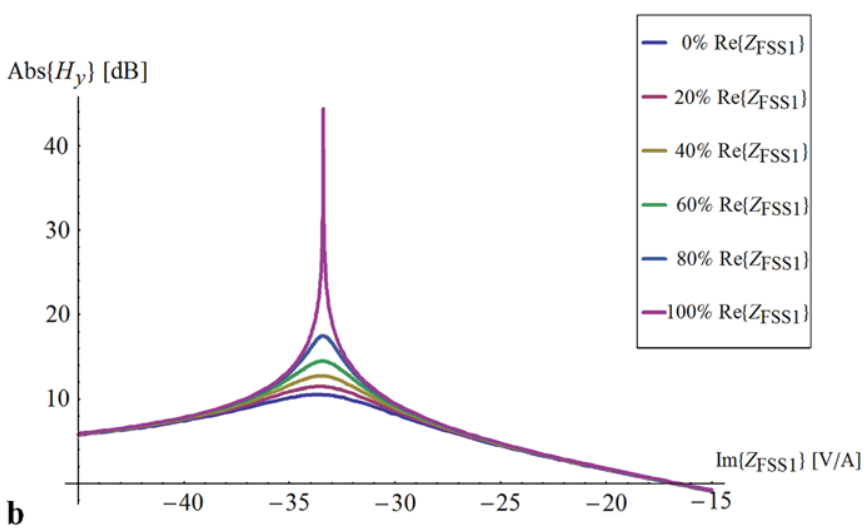

peak of $H_{y}$ is obtained for $Z_{\mathrm{FSS}}=-2.98-j 33.39 \Omega$. (b) Tolerances to a mismatch of the real part moving from the exact design value, $100 \% \operatorname{Re}\left\{Z_{\mathrm{FSS}}\right\}$, to its complete neglection, $0 \% \operatorname{Re}\left\{Z_{\mathrm{FSS}}\right\}$, calculated by the equivalent TL model of Fig. 1
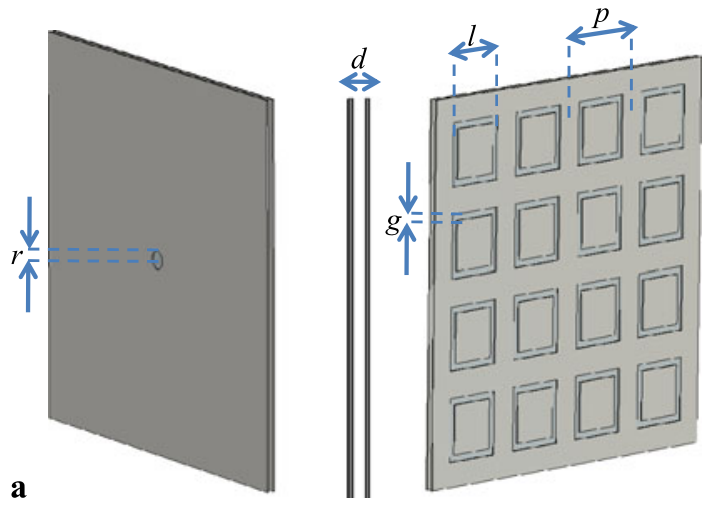

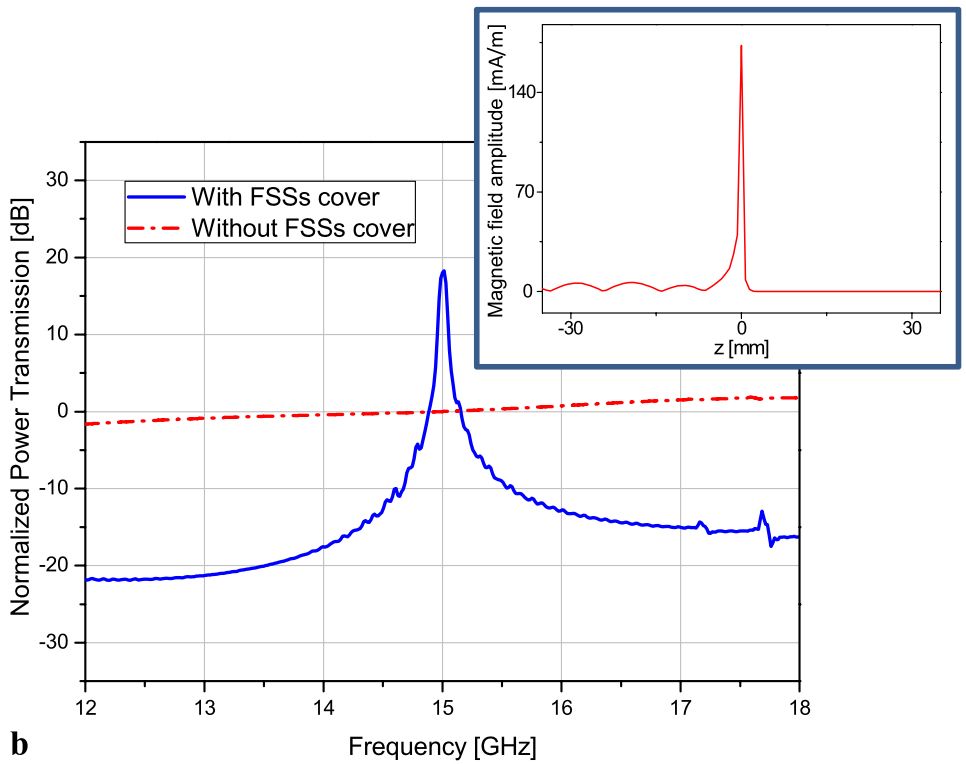

Fig. 3 (a) FSS-based layout. The squared-unit-cell FSS sheet has transverse dimensions $17 \mathrm{~mm} \times 17 \mathrm{~mm}$. The thickness of the copper metallization is $t=0.07 \mathrm{~mm}$. The distance FSS-screen is $d=$ $0.25 \mathrm{~mm}$. The center-center distance between two inner elements for both $x$ and $y$ directions is $p=4 \mathrm{~mm}$. The radius of the hole is $r=0.4 \mathrm{~mm}$. The inner gap $g=0.4 \mathrm{~mm}$. The element side $l=2.9 \mathrm{~mm}$.

Finally, due to the intrinsic symmetry of any setup involving the transmission through an aperture placed on an opaque screen, it is also expected that, pairing two identical structures on both sides of the screen would result, by reciprocity [3], in a significant further increase of the power transmission. By placing an identical FSS as the one in Fig. 3(a) at the exit face of the screen (Fig. 4(a)), we have found the results reported in Fig. 4(b).

The second resonant sheet introduces a split in the enhancement peak, due to the coupling between the two equiv- (b) Power transmission enhancement for the structure depicted in (a) normalized to the case of absence of the FSS structure at the design frequency. (b, up inset) Amplitude of the tangential magnetic field along the normal axis to the hole at $15 \mathrm{GHz}$ in the case of a TEM $(z)$ impinging wave. The hole plane is placed at $z=0 \mathrm{~mm}$. The magnetic field amplitude in $z=0 \mathrm{~mm}$ without the FSS cover is $5 \mathrm{~mA} / \mathrm{m}$

alent resonating circuits, placed at the entrance and exit sides of the hole. As the coupling is rather weak, being the radius of the hole electrically small, the frequency shift results slightly pronounced.

As a final countercheck, we also verified that the transmission enhancement is not caused by an array of periodically spaced holes, due to the introduction of perfect electric and magnetic boundaries in the simulation, but only by the contribution of a single aperture (see Fig. 4(c)). This is an important test, because enhanced transmission can be easily 

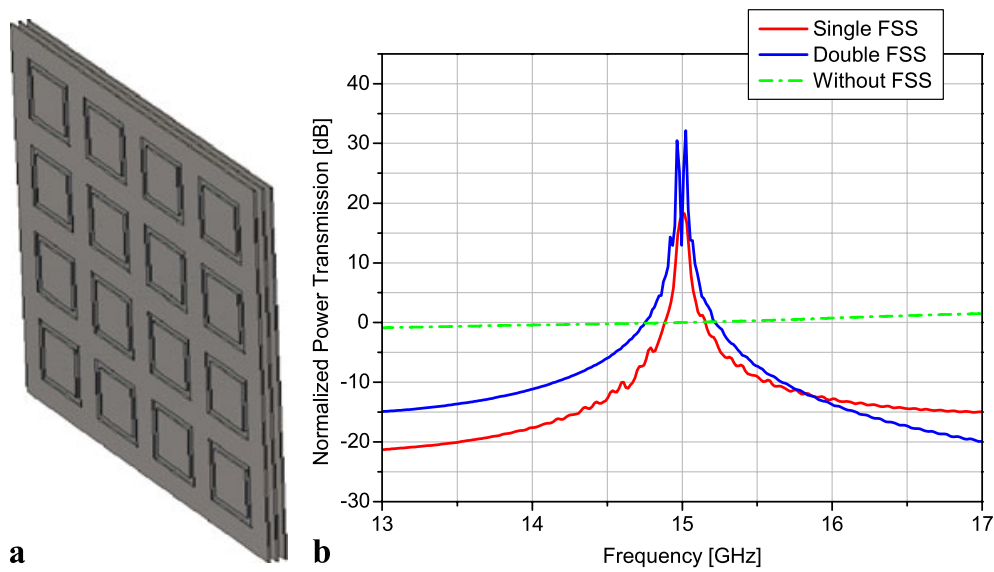

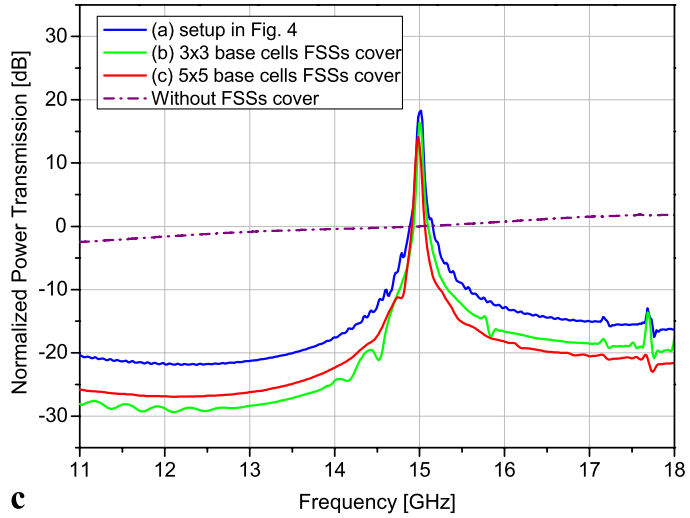

Fig. 4 (a) Geometrical sketch of the symmetrical structure consisting of two identical FSS sheets placed at both sides of the perforated metallic screen. (b) Comparison between the power transmission enhancement of the structure depicted in Fig. 3(a) and the one of (a). (c) Power transmission enhancement for the structure depicted in (a) and for other similar structures with the same base element, periodicity, hole dimensions, and different transverse extensions of the metallic screen that are nine times (b) and twenty-five times (c) bigger than the reference case (a) of Fig. 3(b), respectively

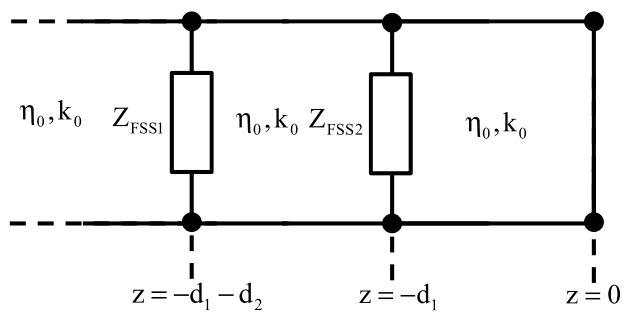

Fig. 5 TL circuit representation of a setup based on a pair of FSSs placed on the left side of the screen. Each FSS sheet is modeled through its surface impedance and the contribution of the hole is again neglected, due to its sub-wavelength dimensions

obtained also from an array of holes, by a proper choice of their periodicity [8].

Finally, we show the effect of using multiple FSS sheets, placed on one side of the screen only. We consider, for instance, the case of two FSS sheets, placed at a distance $d_{1}$ and $d_{1}+d_{2}$ from the metallic plate where the hole is placed. The equivalent TL model for this structure is depicted in Fig. 5.

Assuming a TE $(z)$ polarized electromagnetic field propagating through the structure (the TM case can be obtained again by duality) and both $d_{1}$ and $d_{2}$ electrically small, by imposing the denominator of the magnetic field amplitude to vanish at $z=0$, a solution for $Z_{\mathrm{FSS} 1}$ is found as

$$
\begin{aligned}
& Z_{\mathrm{FSS} 1}^{\mathrm{TE}}=-j k_{0} Z_{0} Z_{\mathrm{FSS} 2}^{\mathrm{TE}} \frac{\left(d_{1}+d_{2}\right)\left(j+2 k_{z} d_{2}\right)}{Z_{\mathrm{FSS} 2}^{\mathrm{TE}}\left(2 k_{z} d_{1}-j\right)+Z_{0} k_{0} d_{1}}, \\
& \left\{\begin{array}{l}
k_{z} d_{1} \ll 1 \\
k_{z} d_{2} \ll 1
\end{array}\right.
\end{aligned}
$$

If the following two additional conditions are introduced:

$\operatorname{Re}\left\{Z_{\mathrm{FSS} 2}^{\mathrm{TE}}\right\}=0, \quad d_{1}=d_{2}$

without any loss in generality, the imaginary and real parts of $Z_{\mathrm{FSS} 1}$ can be, then, evaluated as a function of the imaginary part of $Z_{\mathrm{FSS} 2}$ (see Fig. 6). The final result is that, if the distances of the FSS sheets from the metallic plate are electrically small, it is possible to obtain two simple solutions, one purely capacitive, the other purely inductive:

$Z_{\mathrm{FSS} 2}^{\mathrm{TE}}=-\frac{1}{2} j k_{0} d Z_{0}, \quad Z_{\mathrm{FSS} 1}^{\mathrm{TE}}=2 j k_{0} d Z_{0}$

This configuration exhibits, in principle, better angular performance and tolerance to a mismatch of the real part of the surface impedance compared to the one in which a single capacitive FSS is used $\left(k_{z}\right.$ does not appear in the solution, after a first order expansion of the exact solutions, and both $\operatorname{Re}\left\{Z_{\mathrm{FSS} 2}\right\}$ and $\operatorname{Re}\left\{Z_{\mathrm{FSS} 1}\right\}$ vanish identically). These simple considerations open the doors to more complicated layouts, which can even improve the performance of the setups we have previously proposed in Figs. 3(a) and 4(a). However, the design and the numerical characterization of such structures is out of the scope of this paper and will be the subject of a future publication.

\section{Conclusions}

In this paper, an FSSs-based approach for enhancing the power transmission through a sub-wavelength aperture has been presented. A design formula for the FSS surface impedance has been derived for both TE and TM polarizations of the impinging field, using a TL circuit equivalent model. 
Fig. 6 Real and imaginary parts of $Z_{\mathrm{FSS} 1}^{\mathrm{TE}} t$ as a function of he imaginary part of $Z_{\mathrm{FSS} 2}^{\mathrm{TE}}$ under the assumptions (8). The distances are

$d_{1}=d_{2}=0.25 \mathrm{~mm}$ and the design frequency is $15 \mathrm{GHz}$

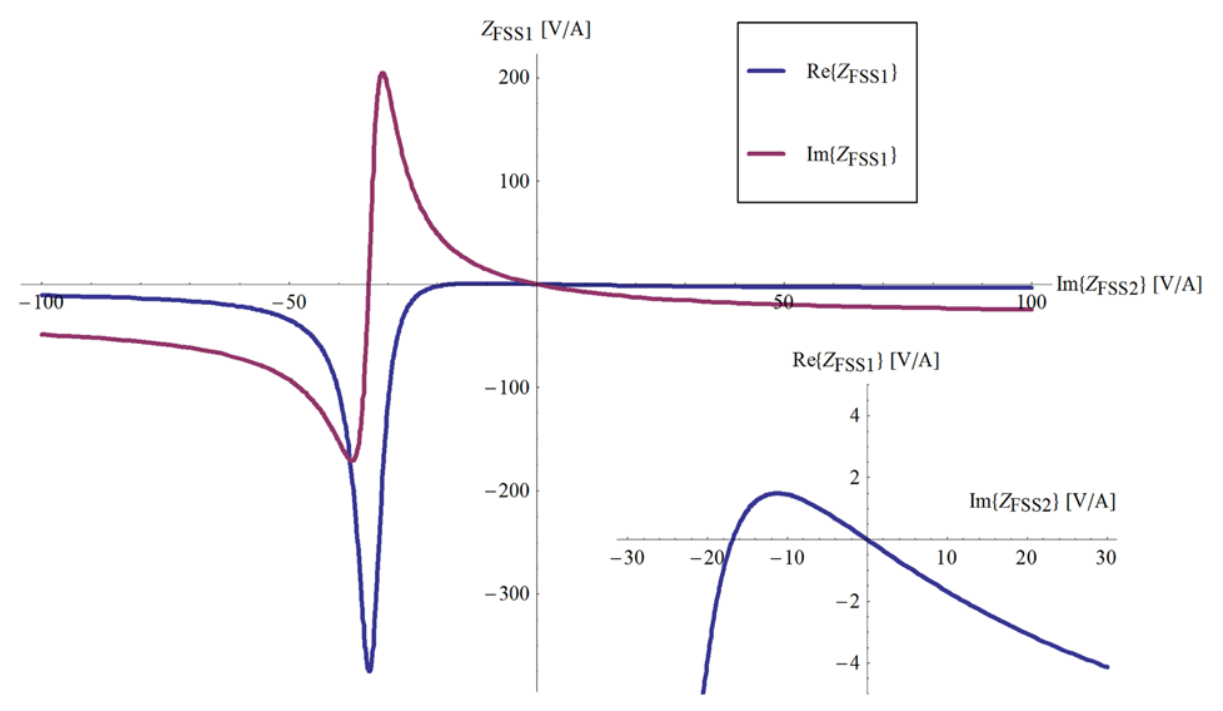

The given analytical models have been validated through proper full-wave simulations performed through CST Studio Suite. Finally, the possibility of using a proper combination of both inductive and capacitive FSSs, placed at one side of the screen, has been investigated, leading to the result that, if the overall thickness of the structure is electrically small, the tolerances to the incidence angle of the impinging radiation and to the real part of the surface impedance can be, in principle, significantly improved.

\section{References}

1. D.E. Grupp, H.J. Lezec, T. Thio, T.W. Ebbsen, Beyond the Bethe limit: tunable enhanced light transmission through a single subwavelength aperture. Adv. Mat. 11(10), 860-862 (1999)
2. A.A. Oliner, D.R. Jackson, Leaky surface-plasmon theory for dramatically enhanced transmission through a sub-wavelength aperture, Part I: Basic features, 2003. IEEE Int. Symp. Antennas Propag. 2, 1091-1094 (2003)

3. A. Alù, F. Bilotti, N. Engheta, L. Vegni, Metamaterial covers over a small aperture. IEEE Trans. Antennas Propag. 54(6), 1632-1643 (2006)

4. H.A. Bethe, Theory of diffraction by small holes. Phys. Rev. 66, 163-182 (1944)

5. M. Caiazzo, S. Maci, N. Engheta, A metamaterial surface for compact cavity resonators. IEEE Antennas Wirel. Propag. Lett. 3(1), 261-264 (2004)

6. B. Munk, Frequency Selective Surfaces: Theory and Design (Wiley, New York, 2000)

7. CST Studio Suite, CST computer simulation technology (2009). http://www.cst.com

8. F.J. García de Abajo, R. Gómez-Medina, J.J. Sáenz, Full transmission through perfect-conductor subwavelength hole arrays. Phys. Rev. E 72(1), 016608 (2005) 